\title{
INDICADORES DE SUSTENTABILIDADE: CONCEITOS, TIPOLOGIAS E APLICAÇÃO AO CONTEXTO DO DESENVOLVIMENTO TURÍSTICO LOCAL
}

\author{
Frederico Yuri Hanai \\ Doutor em Ciências da Eng. Ambiental Escola de Eng. de São Carlos - EESC - USP \\ Professor da Universidade Federal de São Carlos - UFSCAR \\ fredyuri@ufscar.br \\ Evaldo Luiz Gaeta Espíndola \\ Doutor em Ciências da Engenharia Ambiental Universidade de São Paulo - USP \\ Professor da Universidade de São Paulo - USP \\ elgaeta@sc.usp.br
}

\section{RESUMO}

As aspirações da sustentabilidade têm alcançado notável difusão e exigido bases referenciais e instrumentos metodológicos que possibilitem monitorar os processos de desenvolvimento em diversos contextos e situações, com o uso de indicadores. O objetivo do presente trabalho é discorrer sobre as abordagens conceituais de indicadores, suas tipologias, modelos, sistemas e aplicação ao contexto do desenvolvimento do turismo. Os indicadores de sustentabilidade permitem a análise objetiva sobre as condições atuais e situações desejáveis, devendo ser capazes de mostrar tendências ao longo do tempo dos processos de desenvolvimento, inclusive do turismo. Os indicadores de sustentabilidade aplicados ao contexto do desenvolvimento turístico constituem instrumentos práticos para a gestão e o controle da atividade turística numa localidade, sendo sua seleção e monitoramento periódico um componente fundamental para o planejamento e desenvolvimento do turismo sustentável.

Palavras-chave: Indicadores de sustentabilidade; Definição de indicadores; Turismo sustentável; Indicadores de turismo; Sustentabilidade.

\section{INDICATORS OF SUSTAINABILITY: CONCEPTS, TYPOLOGIES AND THEIR APPLICATION IN LOCAL TOURISM DEVELOPMENT}

\begin{abstract}
Expectations concerning sustainability have become notably widespread and call for reference data and methodological instruments that enable the monitoring of development processes in different contexts and situations with the use of indicators. The objective of this study is to address the conceptual framework concerning indicators, their types, the models, systems and applications in the context of tourism development. Sustainability indicators allow for the objective analysis of the current conditions and the desired situations, and they must be able to show trends over the course of developmental processes, including tourism. The indicators of sustainability used in tourism development represent practical instruments for managing and controlling tourism in a particular area, and the choice and frequent monitoring of such indicators are essential for planning and developing sustainable tourism.
\end{abstract}

Keywords: Sustainability indicators; Definition of indicators; Sustainable tourism; Tourism indicators; Sustainability. 


\section{INTRODUÇÃO}

O conceito de "desenvolvimento sustentável" e a ideia de "sustentabilidade" alcançaram notável difusão e vêm sendo interpretados, apropriados e enriquecidos com novas reflexões e diversas abordagens por vários segmentos sociais em distintas situações, estímulos e intenções.

As diversas compreensões e significações relacionadas ao conceito de desenvolvimento sustentável e sustentabilidade conferem uma série de implicações e princípios éticos, que vêm sendo inseridos e aplicados nos contextos acadêmicos, segmentos sociais, planos e processos de desenvolvimento.

Porém, reconhece-se a dificuldade de se medir e avaliar os processos de sustentabilidade do desenvolvimento, pois definitivamente trata-se de compreender uma "complexidade organizada em torno das diversas inter-relações entre os sistemas ambientais e socioeconômicos" (Jiménez Herrero, 2006, p. 8).

Assim, têm surgido diversas propostas de sistemas de medição que buscam identificar os significados de sustentabilidade. Estes significados podem ser identificados por meio de indicadores capazes de refletir e medir, de forma mais objetiva possível, os processos de desenvolvimento sob a égide da sustentabilidade.

Os indicadores "são componentes essenciais na avaliação global do progresso rumo ao desenvolvimento sustentável” (Gallopín, 1997, p. 1). "São componentes que fornecem informações indispensáveis para a compreensão do mundo, para tomada de decisões e para planificação de ações" (Meadows, 1998, p. 1).

Os indicadores identificam as características relevantes de um sistema e clarificam as complexas relações entre as diferentes variáveis envolvidas num fenômeno específico, tornando-o visível ou perceptível para comunicar as suas informações contidas, constituindo-se em instrumentos úteis de análise objetiva sobre o fenômeno considerado. Constituem-se como valiosos e úteis instrumentos para subsidiar o processo de tomada de decisão em gestão, desenvolvimento e monitoramento de projetos e políticas de desenvolvimento sustentável.

Os indicadores surgem de valores e criam valores. "Não somente medimos aquilo que valorizamos, mas também valorizamos aquilo que medimos" (Meadows, 1998, p. 2).

Além do simples processo de medição de dados, os indicadores de sustentabilidade podem estimular o processo para intensificar a ampla compreensão dos problemas sociais e ambientais, facilitar a capacidade da comunidade em criar e conduzir políticas e projetos de desenvolvimento (Reed, Fraser \& Dougill, 2006).

A incorporação dos princípios de sustentabilidade às diversas políticas setoriais de desenvolvimento, dentre elas, a turística, favoreceu a inserção e o uso de indicadores nos instrumentos e técnicas de planejamento, organização e gestão do turismo sustentável.

É possível, com o uso de indicadores, o monitoramento contínuo e consistente de transformações do desenvolvimento do turismo ao longo do tempo numa localidade, podendo visualizar e reforçar metas pré-definidas. Os indicadores servem não apenas para informar sobre a situação do processo de desenvolvimento do turismo, mas permitem também verificar se os objetivos da sustentabilidade estão sendo atingidos.

Desta forma, o objetivo do presente trabalho é discorrer sobre as abordagens conceituais de indicadores, assim como suas tipologias, modelos e sistemas usualmente empregados, visando a sua compreensão e aplicação ao contexto do desenvolvimento sustentável do turismo. 


\section{CONCEITOS E CONSIDERAÇÕES SOBRE INDICADORES}

Os indicadores são variáveis que representam operacionalmente um atributo (qualidade, característica ou propriedade) de um sistema, sintetizando as informações essenciais sobre a sua viabilidade e sua dinâmica de transformação (United Nations Division for Sustainable Development, 2001).

Os indicadores são necessários para informar o estado de um sistema e também para intervir e corrigir o seu direcionamento a determinados objetivos (Gallopín, 1997; Bossel, 1999; United Nations Division for Sustainable Development, 2001). Entretanto, a medição da sustentabilidade não pode ser vista somente de uma situação estática em um momento, mas sim como medição integrada ao longo do tempo para documentar processos, e capazes de mostrar tendências, devido a inerente dimensão temporal inserida no conceito de desenvolvimento sustentável (Gallopín, 1997).

Para Dahl (1997), qualquer abordagem detalhada de indicadores de desenvolvimento sustentável deve incluir tipos de indicadores que contabilizam as múltiplas dimensões interrelacionadas de qualquer sociedade, e novas formas de identificar a sustentabilidade ao longo do tempo.

O uso de indicadores, como medidores de processo do desenvolvimento sustentável, possui as seguintes funções principais (Watson \& Cole, 1992; Gallopín, 1996; Bossel, 1999; Frausto Martínez, Chalé Caamal \& Rojas López, 2005; Reed, Fraser \& Dougill, 2006):

- reconhecer metas e objetivos, mostrando se condições e tendências em relação às finalidades de gestão estão sendo atingidos e satisfeitos;

- fornecer antecipadamente uma informação de advertência, sinalizando a necessidade de ações corretivas da estratégia de gestão;

- subsidiar o processo de tomada de decisão, proporcionando informação relevante para apoiar a implementação de políticas em diferentes níveis da sociedade (bairros, distritos, cidades, países, estados, regiões);

- tornar-se a base para o gerenciamento dos impactos ambientais (avaliar a eficiência de várias alternativas);

- refletir a condição geral de um sistema, permitindo análise comparativa no tempo e no espaço (situações e locais);

- antecipar condições e situações futuras de risco e de conflito;

- orientar projetos e políticas de desenvolvimento.

Os indicadores podem ter objetivos (Romagosa Casals \& Cuétara Sánchez, 2001):

- científicos, para o conhecimento do estado do meio ambiente, da sociedade ou da economia;

- políticos, para o planejamento, gestão e avaliação das políticas aplicadas; e

- informativos, para a comunicação e sensibilização cidadã e dos poderes públicos e econômicos.

O uso de indicadores implica conhecer o que se quer avaliar, selecionar a informação relevante (o excesso de informação desinforma) e por último, sintetizar a informação em uma série de medidas úteis e significativas para os responsáveis pela tomada de decisão (Sancho Pérez, García Mesanat, Pedro Bueno \& Yagüe Perales, 2001).

O desenvolvimento de indicadores, por sua vez, baseia-se num processo de duplo sentido: além de servir aos propósitos e necessidades das políticas locais, é desejável também que auxilie na elaboração e na concretização destas políticas, num amplo processo comunicativo (Valentin \& Spangenberg, 2000).

Deve-se mencionar ainda que os indicadores de sustentabilidade podem ir além do simples processo de medição de dados. Eles podem estimular o processo para intensificar a ampla 
compreensão dos problemas sociais e ambientais, facilitar a capacidade da comunidade em criar e conduzir políticas e projetos de desenvolvimento (Reed et al., 2006).

\section{CARACTERÍSTICAS E PROPRIEDADES DESEJÁVEIS DOS INDICADORES}

As seguintes considerações básicas sobre indicadores influenciam na determinação de suas características e propriedades desejáveis (Sirakaya, Jamal \& Choi , 2000; Miller, 2001; Organization for Economic Co-Operation and Development, 2002a, 2002b; Blázquez, Murray Mas \& Mateu i Lladó, 2002 e Frausto Martínez et al., 2005):

- a pertinência política e interesse público: os indicadores estão relacionados com objetivos políticos e de interesse público para tomada de decisão estratética;

- a disponibilidade de dados, objetividade e exatidão da análise da informação: os indicadores dependem da existência de dados e da possibilidade de mensuração de valores para análise da informação;

- a compreensão e utilidade pública: os indicadores devem propiciar o acesso de manejo, com credibilidade e confiabilidade para os usuários e aptidão para a divulgação da informação;

- a adaptabilidade e comparabilidade no tempo e no espaço: os indicadores devem guiar políticas e decisões nos níveis da sociedade e cobrir toda abrangência dos ambientes socioeconômicos, culturais, naturais e políticos nos níveis local, regional, nacional e internacional;

- a capacidade preditiva: os indicadores devem possibilitar a previsão e a advertência sobre problemas, riscos e mudanças significativas futuras;

- a cobertura de fenômenos sistêmicos e habilidade integrativa: o processo de desenvolvimento de indicadores não pode ser ao acaso, em que requer uma abordagem sistemática para desenvolver indicadores capazes de fornecer uma visão integrada das condições específicas e gerais pertencentes a sustentabilidade.

Estas considerações fundamentais desdobram-se nas seguintes características e propriedades desejáveis e requeridas para aplicação dos indicadores em um sistema (Gallopín, 1997; Romagosa Casals \& Cuétara Sáchez, 2001; Ivars Baidal, 2001; Sancho Pérez et al., 2001; Yunis, 2002; Andreu et al., 2003):

- Validez científica: o indicador deve ter um bom fundamento teórico em termos técnicos e científicos, baseado em um conhecimento consistente do sistema investigado e de seus atributos;

- Simplicidade e compreensão: o indicador deve ser simples e claro, seu significado deve ser de fácil compreensão, também por não especialistas;

- Representatividade e capacidade informativa: a informação que contém o indicador deve ser representativa da condição identificada;

- Sensibilidade a mudanças: o indicador deve sinalizar as mudanças de tendência no ambiente ou nas atividades humanas relacionadas, preferencialmente em curto prazo;

- Confiabilidade dos dados: os dados devem ser os mais confiáveis possíveis e de boa qualidade;

- Relevância: o indicador deve prover informação para os usuários e para determinar objetivos e metas;

- Comparabilidade: o indicador deve ser apresentado de tal forma que permita comparações entre territórios e ao longo de uma série temporal (esta característica

Revista de Gestão Social e Ambiental - RGSA, São Paulo, v. 5, n. 3, p. 135-149, set./dez. 2011. 
amplia elementos de referência, consolidando a aplicação de metodologias de obtenção de dados e generalização do seu uso);

- Validação: o indicador deve possuir consenso e estar baseado em padrões internacionais;

- Predição: o indicador deve prover sinais de alarme prévio sobre as tendências negativas futuras;

- Cobertura geográfica: o indicador deve basear em temas de caráter regional extensíveis a escala nacional e úteis a diferentes níveis de decisões políticas;

- Custo-eficiência: o indicador deve ser eficiente em termos de uma razão custo/benefício razoável para obtenção de dados e para uso da informação;

- Ressonância: o indicador deve possuir a capacidade de despertar interesse dos agentes envolvidos;

- Adaptabilidade: o indicador deve possibilitar a sua adaptação às especificidades locais e temporais (situações).

Pelo exposto, torna-se um grande desafio a consideração de todas estas características e propriedades desejáveis no processo de definição de um sistema de indicadores de sustentabilidade. Além disso, considerações sobre o número adequado e a natureza qualitativa ou quantitativa dos indicadores devem ser discutidas para a compreensão de sua utilidade e aplicação num sistema.

\section{PROCEDIMENTOS PARA SELEÇÃO E DEFINIÇÃO DE INDICADORES}

O International Institute for Sustainable Development (IISD) produziu um conjunto de princípios norteadores para auxiliar e orientar o processo de desenvolvimento sustentável: objetivos; perspectiva holística; equidade e disparidade, condições ecológicas, bem estar humano e social; adequada abrangência no tempo e espaço; foco prático; abertura; comunicação eficiente; e capacidade institucional de gestão. Os princípios servem como diretrizes práticas para o processo de avaliação de sistemas e identificação de indicadores para medição em campo, compilação, interpretação e comunicação dos resultados (Hardi \& Zdan, 1997; Pintér, Hardi \& Bartelmus, 2005).

Para o processo de desenvolvimento de um conjunto de indicadores Bossel (1999) sugere as seguintes etapas:

- compreensão conceitual do sistema como um todo (modelo do sistema, componentes e interações);

- identificação de indicadores representativos (concentrar em variáveis essenciais na seleção de indicadores de viabilidade do sistema);

- avaliação do desempenho dos indicadores (satisfação dos orientadores básicos, que estão descritos a seguir);

- desenvolvimento de um processo participativo (trazer um amplo espectro de conhecimento, experiências, modelos, considerações sociais e ambientais).

\subsection{Natureza qualitativa (subjetiva) e quantitativa (objetiva) dos indicadores}

Segundo Gallopín (1997), um indicador pode ser uma variável qualitativa (nominal), uma variável de classificação (ordinal) ou uma variável quantitativa. Os indicadores qualitativos podem ser preferenciais aos indicadores quantitativos em pelo menos três casos: quando a informação quantitativa não está disponível; quando o atributo de interesse é inerentemente impossível de ser quantificado; e quando o custo das considerações torna-se determinante.

Os indicadores objetivos (quantitativos) medem essencialmente quantidade e são geralmente considerados mais confiáveis e valiosos e certamente de mais fácil comunicação e validação 
(Meadows, 1998). Entretanto, os indicadores de qualidade (que possuem característica subjetiva), apesar de serem mais difíceis de definir e medir, merecem ser considerados.

Existem propósitos vitais que dependem dos indicadores subjetivos e qualitativos. A escolha de direcionar a atenção somente àquilo que é quantitativamente medível é também uma escolha subjetiva, e por isso, não prudente. Todos os indicadores são pelo menos parcialmente subjetivos. A escolha de um indicador é baseada em alguns valores, algumas intenções humanas (que direcionam ao que é importante ser medido) e por isso é inerentemente subjetiva (Meadows, 1998).

O uso combinado de indicadores objetivos e subjetivos pode mitigar as respectivas deficiências de ambos indicadores e fornecer informação melhor e mais precisa para tomadores de decisão criarem um sistema forte de monitoramento que pode melhorar o processo de desenvolvimento. "Uma avaliação utilizando-se ambos indicadores objetivos e subjetivos ajudará a criar fortes e robustos sistemas de monitoramento" (Choi \& Sirakaya, 2006, p. 1286).

\section{ESTRUTURAS E SISTEMAS DE INDICADORES DE SUSTENTABILIDADE E DE DESENVOLVIMENTO SUSTENTÁVEL}

Nos últimos tempos, uma quantidade de indicadores tem surgido na tentativa de medir o progresso em direção ao objetivo da política de desenvolvimento sustentável.

Devido às diversas interpretações do conceito de sustentabilidade, não é de se surpreender que existam muitos tipos, modelos e sistemas diferentes de indicadores de sustentabilidade (Patterson, 2002), que são apresentados resumidamente a seguir.

\subsection{Estrutura das Nações Unidas para o desenvolvimento de estatísticas ambientais (FDES - Framework for the Development of Environment Statistics) \\ Desenvolvida no começo da década de 1980, esta foi a primeira estrutura oficial amplamente reconhecida para a organização de indicadores ambientais. Consiste essencialmente numa lista de verificação destinada a reunir sistematicamente as estatísticas ambientais e os indicadores, porém sem a tentativa de estabelecer contabilização ou relações funcionais entre as variáveis estatísticas e os indicadores (Gallopín, 1997; Patterson, 2002).}

\subsection{Modelo Pressão-Estado-Resposta (PER, em inglês PSR - Pressure-State-Response) e Força Motriz (Condutora)-Estado-Resposta (FCER, em inglês DPSIR - Driving Forces-Pressure- State-Impact-Response)}

Conhecido internacionalmente, este modelo é talvez o sistema mais amplamente utilizado para indicadores de sustentabilidade e foi proposto por um grupo de pesquisadores canadenses no começo dos anos 1990 (Organization for Economic Co-Operation and Development, 2002a, 2002b). O modelo foi adotado pela OECD (Organization for Economic and Cooperation Development) e pela Comissão das Nações Unidas para o Desenvolvimento Sustentável (United Nations Comission on Sustainable Development - UNCSD) como estrutura padrão para indicadores de sustentabilidade.

A partir do modelo PER, surgiu o modelo Força Motriz (Condutora)- Estado-Resposta (FCER, em inglês DPSIR - Driving Forces-Pressure-State-Impact-Response), que inclui similarmente os aspectos sociais, econômicos e ambientais, incorporando as causas da pressão e dos impactos produzidos no estado das condições ambientais.

O modelo PER é cíclico e se baseia no conceito de casualidade, e reflete as relações que as atividades humanas (pressões, forças motrizes) exercem sobre o meio ambiente e modificam a qualidade e quantidade (estado) dos recursos naturais e das situações sociais e econômicas. A sociedade responde a estas mudanças por meio de políticas ambientais, econômicas e setoriais 
(respostas), e estas produzem retroalimentação direcionada a modificar as pressões das atividades humanas (Organization for Economic Co-Operation and Development, 2002a, 2002b).

O modelo se organiza em três tipos básicos de indicadores:

- Indicadores de pressão, que descrevem refletem as pressões diretas e indiretas das atividades humanas exercidas sobre o meio ambiente;

- Indicadores de estado, que descrevem as condições ambientais (qualidade do meio ambiente, quantidade e qualidade dos recursos naturais) num determinado momento;

- Indicadores de resposta, que correspondem aos esforços que a sociedade responde às mudanças ambientais. Integram as políticas das diferentes administrações territoriais e setoriais para prevenir, atenuar ou reduzir a degradação das condições ambientais.

Para Bossel (1999), Marzall e Almeida (1999), a mais séria objeção a esta abordagem sobre indicadores é a que o modelo negligencia a natureza sistêmica e dinâmica do processo.

O modelo PER/FCER é baseado na casualidade e considera a lógica linear (efeito em função da causa), e não a avaliação de sua interação e por isso tem conduzido a políticas institucionais errôneas devido a uma interpretação simplificada das inter-relações entre os aspectos a serem medidos (Gallopín, 1997; Segnestam, 1999; Sánchez Rubio, 2004; Frausto Martínez et al., 2005; Pintér et al., 2005).

\subsection{Indicadores para Agenda 21 e sustentabilidade local}

Nesta abordagem, os indicadores são gerados por meio de processos da construção da Agenda 21 local, cuja ênfase está no conceito de sustentabilidade local com o estabelecimento de indicadores participativos de desenvolvimento sustentável.

A definição de indicadores sugere um processo de consulta e de consenso que discutem problemas específicos de uma localidade em direção ao desenvolvimento sustentável.

As iniciativas de estabelecimento de indicadores participativos têm princípio de trabalhar e definir indicadores relacionados diretamente aos objetivos e metas que conduzam à sustentabilidade local. Entretanto, existem limitações específicas da aplicação deste modelo, sendo a principal relacionada com a perda de visão regional, estando integrada e influenciada por processos de tomadas de decisões regionais e nacionais (United Nations Division for Sustainable Development, 2001; Frausto Martínez et al., 2005).

\subsection{Indicadores de desenvolvimento sustentável propostos pelo IBGE (Instituto Brasileiro de Geografia e Estatística) - Brasil}

No Brasil, o trabalho de construção de Indicadores de Desenvolvimento Sustentável realizado pelo IBGE foi inspirado nos trabalhos desenvolvidos pela Comissão para o Desenvolvimento Sustentável das Nações Unidas, que publicou o documento Indicators of sustainable development: framework and methodologies (Commission on Sustainable Development, 2001).

O trabalho do IBGE toma como referência este documento e as recomendações adicionais que o sucederam, adaptando seu conteúdo às particularidades brasileiras. O conjunto de indicadores selecionados representa 59 indicadores distribuídos nas seguintes dimensões e seus respectivos temas: ambiental (atmosfera, terra, água doce, oceanos, mares, áreas costeiras, biodiversidade, saneamento), social (população, trabalho, rendimento, saúde, educação, habitação, segurança), econômica (quadro econômico, padrões de produção e consumo) e institucional (quadro institucional e capacidade institucional) (Instituto Brasileiro de Geografia e Estatística, 2004). 


\subsection{Alguns indicadores agregados e índices}

A seguir, são listados alguns indicadores e índices compostos usualmente empregados na avaliação de processos de desenvolvimento sustentável:

- Pegada Ecológica (Ecological Footprint): é um indicador de sustentabilidade baseado em critérios ecológicos e foi desenvolvida e proposta por Wackernagel e Rees (1996) com objetivo de estimar a área requerida para sustentar direta e indiretamente a atividade de uma população ou de um sistema econômico. Corresponde, portanto, à quantidade de área terrestre e de água de um ecossistema requerida para manter o padrão de vida de uma população pelo uso e consumo dos recursos materiais e energéticos, assim como para a assimilação dos resíduos produzidos (Wackernagel \& Rees, 1996);

- $\quad$ Painel de Sustentabilidade - Dashboard of Sustainability: é um índice agregado de vários indicadores que emprega meios visuais de apresentação para mostrar as dimensões primárias da sustentabilidade, fornecendo informações quantitativas e qualitativas sobre o progresso em direção à sustentabilidade. A ferramenta utiliza um painel com três mostradores que representam a sustentabilidade do sistema no que se refere às dimensões propostas e é usado para a comparação entre nações, regiões e cidades (Van Bellen, 2004);

- Barômetro da sustentabilidade (Barometer of Sustainability): é uma ferramenta para medir e comunicar o bem estar de uma sociedade e o progresso em direção ao desenvolvimento sustentável por meio da integração de indicadores biofísicos e de saúde social. A ferramenta de avaliação é uma combinação do bem-estar humano e do ecossistema, sendo que cada um deles é mensurado individualmente por seus respectivos índices, representados graficamente na forma bidimensional, indicando a situação em relação à sustentabilidade (Prescott-Allen, 1997);

- Indicadores de Fluxo de Material: o Instituto Wuppertal na Alemanha tem desenvolvido indicadores que descrevem sistematicamente os fluxos físicos de recursos naturais, contabilizando todos os materiais e energia (incluindo as perdas) utilizados num processo produtivo (extração, produção, fabricação, uso, reciclagem e disposição final) de um produto particular ou serviço (Patterson, 2002; Ness, Urbel-Piirsalu, Anderberg, Olsson, 2007);

- Indicadores de Análise do Ciclo de Vida: a Análise do Ciclo de Vida (ACV) é uma ferramenta de análise sistêmica e holística de impactos ambientais, que quantifica os fluxos de energia e de materiais durante todo o ciclo de vida de produtos ou serviços (extração, processamento, manufatura, transporte, distribuição, uso, reuso, reciclagem e disposição final), permitindo acompanhamento dos ciclos de produção e a identificação de alternativas de melhoria e interação entre processos produtivos (Chehebe, 1997; Patterson, 2002; Ness et al., 2007);

- Indicadores do Método Ameba: este modelo está inserido no conjunto de indicadores que buscam medir a sustentabilidade em termos de critérios ecológicos, e foi desenvolvida por pesquisadores holandeses e aplicadas em ecossistemas aquáticos nos Países Baixos e utiliza um número de indicadores variáveis dispostos graficamente para definir a garantia sustentável do ecossistema (Patterson, 2002);

- Índice de sustentabilidade ambiental (ESI - Environmental Sustainability Index): este índice foi desenvolvido pelo Fórum Mundial de Economia no ano de 2001 para medir o progresso em direção a sustentabilidade ambiental e quantifica a probabilidade que um território possui de preservar valiosos recursos naturais efetivamente ao longo de um período de tempo (Patterson, 2002; Ness et al., 2007); 
- Living Planet Index (LPI): idealizado e utilizado pelo World Wildlife Fund (WWF), este índice mede o estado e a tendência global da biodiversidade em ecossistemas florestais, espécies marinhas e de águas doces (Patterson, 2002);

- Índice de Pressão de Consumo: é designado para medir a pressão que uma nação individualmente exerce sobre ecossistemas naturais, baseada no consumo de recursos e emissão da poluição (Patterson, 2002);

- Índice de Desenvolvimento Humano (IDH): o IDH foi desenvolvido nos anos noventa (United Nations Development Programme [UNDP], 1990) e mede as dimensões sociais e econômicas do desenvolvimento sustentável baseando-se nos parâmetros de longevidade (expectativa de vida), de educação (taxa de analfabetismo adulta e matrículas escolares) e do padrão de vida (Produto Interno Bruto per capita) (Patterson, 2002);

- Índice de Desempenho Ambiental (EPI - Environmental Performance Index): consiste na medida padrão do desempenho de políticas direcionadas à redução de estresses ambientais na saúde humana e promoção da vitalidade de ecossistema e gestão de recursos naturais (Böhringer \& Jochem, 2007);

- Índice de Vulnerabilidade Ambiental (EVI - Environmental Vulnerability Index): compreende 32 indicadores de riscos, 8 indicadores de resistência e 10 indicadores que medem os danos e vulnerabilidade do ambiente (Böhringer \& Jochem, 2007);

- Índice de bem-estar econômico sustentável (ISEW - Index of Sustainable Economic Welfare): este índice foi desenvolvido por Daly e Cobb em 1989 para integrar exterioridades econômicas e ambientais no cômputo do bem-estar nacional (Ness et al., 2007);

- Índice de bem-estar (WI - Well-Being Index): consiste de dois índices: o índice de bemestar humano e o índice de bem-estar do ecossistema, que agregam mais de 60 diferentes indicadores (Ness et al., 2007).

\section{INDICADORES DE SUSTENTABILIDADE E SUA APLICAÇÃO NO CONTEXTO DO DESENVOLVIMENTO TURÍSTICO}

Os indicadores de sustentabilidade no desenvolvimento turístico constituem instrumentos práticos para o planejamento, implantação e controle da atividade turística, ao proporcionar informação adequada e confiável das condições ambientais, econômicas e sociais do turismo (Medina Muñoz \& Medina Muñoz, 2003).

Os indicadores medem o cumprimento de objetivos, metas e desempenhos, proporcionando um sinal preventivo de alerta para a necessidade de mudanças de políticas ou de novas ações, servindo de base para o planejamento e a gestão do turismo (United Nations Environment Programe/World Tourism Organization, 2005).

O indicador é um instrumento que facilita a análise e a avaliação da informação coletada de forma que, conjuntamente com outros instrumentos, permite aos tomadores de decisão reduzir a possibilidade de adotar inadvertidamente decisões desafortunadas (Organización Mundial del Turismo, 2005).

A utilização de indicadores não é um procedimento trivial, se é desejável obter informações reais e claras da situação de um destino turístico e utilizá-las como ferramenta de gestão destes destinos (Sancho Pérez \& García Mesanat, 2006a e 2006b). O uso de indicadores é parte de uma abordagem holística do planejamento e gestão de destinos turísticos, projetado para promover a integração entre comunidades, recursos e setores envolvidos com o turismo (Manning, 1999).

Os indicadores podem ter uma influência determinante no processo de desenvolvimento sustentável do turismo de três formas principais: por meio da informação que geram; a mobilização que criam (associações e envolvimento setorial); e as ações que promovem. Os indicadores marcam sua influência inclusive antes de estarem plenamente desenvolvidos, já que na fase de sua 
elaboração se exploram novos conceitos e se produzem novos conhecimentos sobre o desenvolvimento do turismo sustentável (Organización Mundial del Turismo, 2005).

Os indicadores de sustentabilidade diferem dos tradicionais indicadores de desenvolvimento por levar em consideração a rede complexa de inter-relações e interdependências entre os recursos naturais e culturais e a sociedade local envolvida no sistema turístico (Sirakaya et al., 2001; Choi \& Sirakaya, 2006).

Os indicadores referentes ao desenvolvimento do turismo sustentável correspondem ao conjunto de informações formalmente selecionadas que se utiliza regularmente para a medição das mudanças ocasionadas pelo desenvolvimento e gestão das atividades turísticas numa localidade e servem como instrumento para detectar as repercussões nas estruturas turísticas e nos fatores externos que influenciam o turismo (Organización Mundial del Turismo, 2005).

Assim, a seleção de indicadores e seu monitoramento periódico é um componente fundamental para a gestão e o desenvolvimento de atividades turísticas em uma localidade.

Os principais tipos de indicadores (baseados no modelo Pressão-Estado-Resposta, descrito anteriormente) que têm sido utilizados na prática atualmente são (Popova, 2003):

- Indicadores preventivos: auxiliam a identificar e prevenir problemas;

- Indicadores de capacidade de carga ou de medição do estresse: medem fatores externos ambientais e tendências;

- Indicadores de medição do estado dos recursos naturais e volume de demanda dos recursos: auxiliam gestores a identificar quais as mudanças ocorridas nos recursos naturais e suas relações;

- Indicadores de impactos e efeitos do turismo: podem ser integrados a planos de negócios ou serem utilizados para definir ações;

- Indicadores de medição de esforços e ações de gestão: fornecem informações úteis para gestão e obtenção de resultados;

- Indicadores de medição das consequências do manejo: identificam o efeito das ações tomadas para o desenvolvimento sustentável do turismo na comunidade.

Os indicadores de sustentabilidade precisam ser incluídos no monitoramento e gestão de todas as formas de desenvolvimento turístico. Estes indicadores devem envolver aspectos ambientais, tecnológicos, sociais, econômicos e políticos e devem ser planejados e implementados em todos os níveis: local, regional, nacional e internacional (Sirakaya et al., 2001).

\subsection{Indicadores para o desenvolvimento do turismo local}

Atualmente, muitas organizações nacionais e internacionais têm desenvolvido indicadores de sustentabilidade, dentre elas: Organização Mundial de Turismo (OMT); Banco Mundial; Bird Life; International Institute of Sustainable Developmento (IISD); Sustainable Seatle; Instituições das Nações Unidas, tais como United Nations Commission of Sustainable Development (UNCSD) e United Nations Development Program (UNDP). Porém, os esforços empenhados por estas organizações e instituições têm focado no desenvolvimento geral no nível macro, e a maioria destes indicadores não podem ser aplicados para monitorar o desenvolvimento turístico local (Choi \& Sirakaya, 2006).

Os indicadores nacionais e internacionais de turismo sustentável são, necessariamente, amplos e não podem adequar-se plenamente aos distintos tipos de destinos locais (James, 2004). Devido à grande variedade de destinos, é necessário que seja estabelecida uma série de indicadores de turismo local sustentável, vinculados a políticas locais claras e resultados estratégicos.

A diversidade de culturas do mundo e de valores de sistema fornece muitas perspectivas diferentes, cada qual pode ser válida em seu próprio contexto particular. Pode ser perigoso o uso de indicadores internacionalmente para fazer julgamento de valores sobre o conteúdo de 
desenvolvimento. Por esta razão, o conceito de indicadores internacionais de desempenho para o desenvolvimento sustentável é politicamente inaceitável (Dahl, 1997).

A pertinência dos indicadores varia de acordo com as regiões e seu contexto. Os indicadores ambientais devem ser estabelecidos pelas autoridades locais com base nos objetivos de desenvolvimento turístico local. Devem ser remanejados e interpretados dentro do contexto apropriado, levando-se em conta as características ecológicas, geográficas, sociais, econômicas e institucionais de cada região (Organization for Economic Co-Operation and Development, 2002a, 2002b).

Twining-Ward e Butler (2002) argumentam que os indicadores precisam refletir o espaço e o tempo específicos no contexto de uma localidade para que as ferramentas de gestão do turismo sustentável sejam realmente efetivas.

Assim, os indicadores de sustentabilidade podem e devem efetivamente contribuir para o processo de desenvolvimento que combine com as prioridades locais e considere os interesses dos moradores locais (Reed et al., 2006).

Como um indicador descreve um processo específico de controle (e não uma informação exclusivamente numérica), o indicador está fortemente vinculado aos processos particulares dos quais formam parte e deve ser sensível às percepções dos agentes envolvidos em seu desenvolvimento, buscando um sistema que faça com que os resultados sejam facilmente interpretáveis (Sancho Pérez \& García Mesanat, 2006a e 2006b).

A concretização do equilíbrio entre as dimensões ambiental, econômica e sociocultural da sustentabilidade supõe uma revalorização da escala local de planejamento e gestão de turismo. Na escala local, os diagnósticos podem ser mais precisos e a definição da estratégia turística pode ser objeto de consenso mediante a participação da sociedade local (Vera Rebollo \& Ivars Baidal, 2004).

$\mathrm{Na}$ escala local, os indicadores setoriais turísticos devem integrar-se em um sistema de informação territorial global junto com outras variáveis de tipo ambiental ou socioeconômico, já que a ótica exclusivamente setorial do desenvolvimento é inconcebível, inclusive nas áreas de alta especialização turística (Ivars Baidal, 2001).

Ainda assim, os sistemas locais de indicadores de desenvolvimento turístico sustentável devem complementar-se com outras escalas que permitam uma melhor aproximação dos efeitos globais e regionais do turismo (Ivars Baidal, 2001).

\section{CONSIDERAÇÕES FINAIS}

Os indicadores constituem-se como valiosos e úteis instrumentos para subsidiar o processo de tomada de decisão na gestão e no desenvolvimento de projetos e políticas de desenvolvimento. Permitem a análise objetiva sobre as condições atuais e situações desejáveis e devem ser capazes de mostrar tendências ao longo do tempo dos processos de desenvolvimento, reconhecendo metas e objetivos.

A definição de um conjunto apropriado de indicadores para monitoramento do desenvolvimento sustentável não é uma tarefa simples, pois exige selecionar indicadores que representem os relevantes aspectos de viabilidade, sustentabilidade e desempenho do sistema e incorporá-los num procedimento sistemático, objetivo, compacto e compreensível.

Os objetivos do desenvolvimento sustentável do turismo devem considerar o âmbito local como o nível básico e adequado para a aplicação dos indicadores de sustentabilidade, a fim de tornar exequíveis e alcançáveis as características, prioridades desejáveis e interesses específicos locais.

Em função do seu sistema de medição, os indicadores podem ser quantitativos (números simples, proporções, porcentagens) e/ou qualitativos (categorias, níveis de satisfação, etc.), devendo referir-se a variáveis de caráter quantitativo, mas também de caráter qualitativo. Assim, uma 
combinação entre as duas concepções de indicadores (qualitativo e quantitativo) é adequada, pertinente, desejável e enriquecedora para o estabelecimento de um bom sistema de monitoramento.

Os indicadores de sustentabilidade aplicados ao contexto do desenvolvimento turístico constituem instrumentos para a gestão e o controle da atividade turística de um destino, sendo sua seleção e monitoramento periódico componente fundamental para o planejamento e o desenvolvimento local.

\section{AGRADECIMENTOS}

- Ao Programa de Pós-Graduação em Ciências da Engenharia Ambiental (PPG-SEA) e ao Centro de Recursos Hídricos e Ecologia Aplicada (CRHEA) da Escola de Engenharia de São Carlos (EESC) da Universidade de São Paulo (USP) pela disponibilização de recursos para tradução do artigo para a língua inglesa;

- Ao Conselho Nacional de Desenvolvimento Científico e Tecnológico (CNPq) pela concessão da bolsa de doutorado, viabilizando a dedicação integral e exclusiva à realização da presente pesquisa;

- Ao Programa Petrobras Ambiental pelo financiamento do Projeto Mogi-Guaçu que propiciou a realização do presente trabalho;

- Ao Programa Santander Banespa de Mobilidade Internacional de Pós-Graduandos, que financiou o estágio de doutorado na Espanha (Universidade de Vigo).

\section{REFERÊNCIAS}

Andreu, N.; Blázquez, M.; Mateu, J.; López, S.; Mas, L.; Morell, F. et al. (2003). La mesura de la sostenibilitat del turismo a les Illes Balears. Illes Balears: Centre d'Investigació i Tecnologies Turístiques de les Illes Balears (CITTIB).

Blázquez, M.; Murray Mas, I.; Mateu I Lladó, J. (2002). El uso de indicadores de sostenibilidad del turismo para el análisis de la construcción social del espacio. Informe del Seminário Internacional Indicadores Ambientales de Turismo, Murcia (España), 108-113.

Böhringer, C.; Jochem, P.E.P. (2007). Measuring the immeasurable: a survey os sustainable indices. Ecological Economics, 63, pp. 1-8.

Bossel, H. (1999). Indicators for sustainable development: theory, methods, applications: a report to Balaton Group. International Institute for Sustainable Development. Winnipeg, Manitoba, Canada, IISD.

Chehebe, J.R. (1997). Análise do ciclo de vida de produtos: ferramenta gerencial da ISO 14000. Rio de Janeiro: Qulitymark.

Choi, H.C.; Sirakaya, E. (2006). Sustainability indicators for managing community tourism. Tourism Management, 27, 1274-1289.

Commission on Sustainable Development. (2001). Indicators of sustainable development: framework and methodologies. New York: Department of Economic and Social Affairs (Background paper $\mathrm{N}^{\mathrm{o}} 3$ ). 
Dahl, A.L. (1997). The big picture: comprehensive approaches. In: Moldan, B.; Bilharz, S.; Matravers, R. (Ed.), Sustainability indicators: a report on the project on indicators of sustainable development (pp.69-83). Chichester, GB: Wiley and sons.

Frausto Martínez, O.; Chalé Caamal, G.G.; Rojas López, J. (2005). Herramientas y técnicas para la aplicación y evaluación de indicadores de desarrollo sustentable para localidades o municípios. In: Palafoz Muñoz, A. (Ed.), Turismo: teoría y praxis (pp.85-91). Quintana: Universidad de Quintana Roo.

Gallopín, G C. (1996). Environmental and sustainability indicators and the concept of situational indicators: a system approach. Environmental Modeling \& Assessment, 1(3), 101-117.

Gallopín, G.C. (1997). Indicators and their use: information for decision making. In: Moldan, B.; Bilharz, S.; Matravers, R. (Ed.), Sustainability indicators: a report on the project on indicators of sustainable development (pp.13-27). Chichester, GB: Wiley and sons.

Hardi, P.; Zdan, T. (1997). Assessing Sustainable Development: principles in practice. Winnipeg (Canada): International Institute for Sustainable Development.

Instituto Brasileiro de Geografia e Estatística. (2004). Indicadores de desenvolvimento sustentável. Rio de Janeiro: Ministério do Planejamento, Orçamento e Gestão. (Estudos e Pesquisas, Informações Geográfias No 4).

Ivars Baidal, J.A. (2001). Planificación y gestión del desarrollo turístico sostenible: propuestas para la creación de un sistema de indicadores (Proyecto METASIG). Alicante: Universidad de Alicante; Instituto Universitario de Geografia.

James, D. (2004). Indicadores locales de turismo sostenible. Revista estudios turísticos, 161-162, 229-241.

Jiménez Herrero, L.M. (2006, Nov). Los procesos de sostenibilidad en España. Ambienta, pp.8-19.

Manning, T. (1999). Indicators of tourism sustainability. Tourism Management, 20(2), 179-181.

Marzall; K.; Almeida, J. (1999). O estado da arte sobre indicadores de sustentabilidade para agroecossistemas. Atas do Seminário Internacional sobre Potencialidades e Limites do Desenvolvimento Sustentável, Santa Maria-RS, Universidade Federal de Santa Maria, 1-10.

Meadows, D. (1998). Indicators and information systems for sustainable development: a report to the Balaton Group. Hartland: The Sustainability Institute.

Medina Nuñoz, R.D.; Medina Muñoz D.R. (2003). Indicadores del desarrollo sostenible del turismo: una aplicación al caso de Canarias como destino turístico. Congreso de Turismo, Universidad y Empresa. La calidad integral del turismo (pp.289-306). Valencia (España): Fundació Universitat Empresa.

Miller, G. (2001). The development of indicators for sustainable tourism: results of a Delphi survey of tourism researchers. Tourism Management, 22, 351-362.

Ness, B.; Urbel-Piirsalu, E.; Anderberg, S.; Olsson, L. (2007). Categorising tools for sustainable assessment. Ecological Economics, 60, 498-508. 
Organization for Economic Co-Operation and Development. (2002a). Rumo ao desenvolvimento sustentável: indicadores ambientais (Teles, A.N.S.F., Tradução). Salvador: Centro de Recursos Ambientais.

Organization for Economic Co-Operation and Development. (2002b). Working together towards sustainable development: the OECD experience. Paris, France: OECD Publications Service,.

Organización Mundial del Turismo. (2005). Indicadores de desarrollo sostenible para los destinos turísticos: guía práctica. Madrid: OMT.

Patterson, M (2002). Headline indicators for tracking progress to sustainability in New Zealand. Wellington, New Zealand: Ministry for the Environment. (Technical Report $\mathrm{N}^{\mathrm{o}} 71$ ).

Pintér, L.; Hardi, P; Bartelmus, P. (2005). Sustainable development indicators: proposals for a way forward. New York: United Nations Division for Sustainable Development.

Popova, N. (2003). Ecotourism impact/success indicators baseline data 2002:. Sofia, Bulgaria: Biodiversity Conservation \& Economic Growth Projetc.

Prescott-Allen, R. (1997). Barometer of stability. In: Moldan, B.; Bilharz, S.; Matravers, R. Sustainability indicators: a report on the project on indicators of sustainable development (pp.133137). Chichester, GB: Wiley and sons.

Reed, J.S.; Fraser, E.D.G.; Dougill, A.J. (2006). An adaptative learning process for developing and applying sustainability indicators with local communities. Ecological Economics, 59, 406-418.

Romagosa Casals, F.; Cuétara Sánchez, L. (2001). El desarrollo sostenible en destinos turísticos: propuesta de un sistema de indicadores de sostenibilidad. Papers de Turismo, 30, 125-137.

Sánchez Rubio, C.J. (2004). Sistemas de indicadores de sostenibilidad y desarrollo turístico: aplicación a la comarca del Bajo Segura (Alicante). Investigaciones Geográficas, 33, 17-38.

Sancho Pérez, A.; García Mesanat, G. (2006a). ¿Qué indica un indicador? Análisis comparativo en los destinos turísticos. Revista de análisis turístico, 2, 69-75.

Sancho Pérez, A.; García Mesanat, G. (2006b). El papel de los indicadores en la planificación turística. In Actas del Congreso Nacional Turismo y Tecnologías de la Información y las Comunicaciones (pp.1-13). Málaga (España): Escola Universitária de Turismo, Universidad de Málaga.

Sancho Pérez, A.; García Mesanat, G.; Pedro Bueno, A; Yagüe Perales, R.M. (2001). Auditoria de sostenibilidad en los destinos turísticos. Valencia: Instituto de Economia Internacional.

Segnestam, L. (1999). Environmental performance indicators: a second edition note. Washington: World Bank Environment Department. (Environmental Economics Series, paper No 71).

Sirakaya, E.; Jamal, T.B.; Choi, H.S. (2001). Developing indicators for destination sustainability. In: Weaver, D.B (Ed.), The encyclopedia of Ecotourism (pp.411-432). Oxon: CABI. 
Twining-Ward, L.; Butler, R. (2002). Implementing STD on a Small Island: development and use of sustainable tourism development indicators in Samoa. Journal of Sustainable Tourism, 10 (5), 363-387.

United Nations Division for Sustainable Development. (2001). Indicators of sustainable development: guidelines and methodologies. New York: Commission on Sustainable Development.

United Nations Environment Programe/World Tourism Organization. (2005). Making tourism more sustainable: a guide for policy makers. Madrid, Spain: UNEP/WTO.

Valentin, A. Spangenberg, J.H. (2000). A Guide to community sustainability indicators. Environmental Impact Assessment Review, 20, 381-392.

Van Bellen, H.M. (2004). Desenvolvimento sustentável: uma descrição das principais ferramentas de avaliação. Ambiente \& Sociedade, 7(1), 67-87.

Vera Rebollo, J.F.; Ivars Baidal, J.A. (2004). Indicadores de sostenibilidad para destinos maduros: balance y propuestas de aplicación. Madrid: Organización Mundial de Turismo.

Wackernagel, M.; Rees, W. (1996). Our Ecological Footprint: reducing human impact on the Earth. Gabriola Island, BC: New Society.

Watson, A.; Cole, D. (1992). LAC Indicators: an evaluation of progress and list of proposed indicators. In Merigliano, L. (Ed.), Ideas for limits of acceptable change process (pp.65-84). Washington, DC: U.S. Department of Agriculture, Forest Service.

Yunis, E. (2002). Indicadores de turismo sostenible: el enfoque de la OMT. Informe del Seminário Internacional Indicadores Ambientales de Turismo, Murcia (España), 11 - 13.

Data do recebimento do artigo: 17/01/2011

Data do aceite de publicação: 25/11/2011 